\title{
Humane Euthanasia Procedures For Sick, Injured, and/or Debilitated Livestock
}

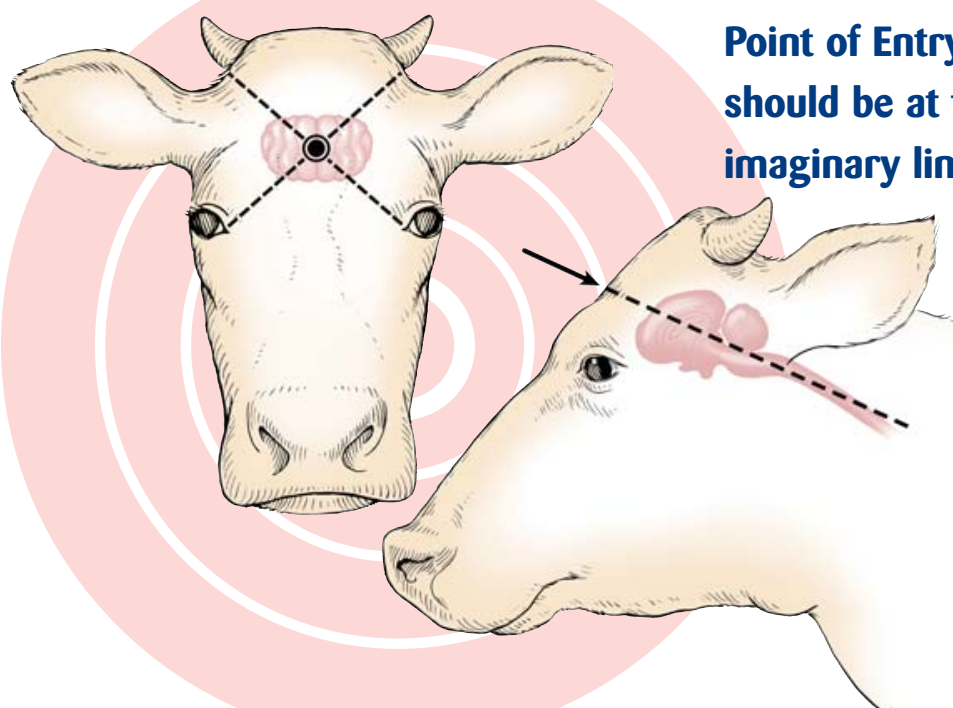

the inside corner of the eye to the base of the opposite horn (or to a point slightly above the opposite ear in a cow without horns). Center of forehead, but NOT BETWEEN THE EYES!

\section{Not Between the Eyes!}


GunshoT Hold firearm perpendicular to the skull and within inches of the intended target when possible. A .22 caliber hollow or soft point bullet is sufficient for young animals. However, larger adult animals require at least a .22 magnum solid point bullet or preferably a $9 \mathrm{~mm}$ or .357 caliber bullet. Proper placement of the bullet is essential and best achieved by holding the firearm within a few inches of the intended target. The firearm should not be held or placed against the head.

Penetrating Captive Bolt Restrain animal so that the device can be held firmly against the skull over the intended site. As described for gunshot, the penetrating captive bolt should be positioned perpendicular to the skull. Always insure death by exsanguination (bleeding out).

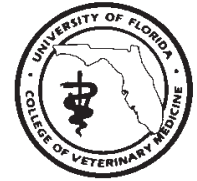

College of Veterinary Medicine/ University of Florida /IFAS www.vetmed.ufl.edu/lacs/HumaneEuthanasia.htm

UF FLIORIDA

IFAS Extension 